\author{
Military Technical College \\ Kobry El-Kobbah, \\ Cairo, Egypt.
}

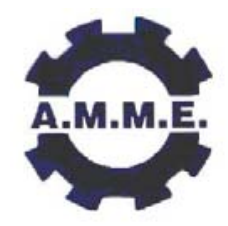
$13^{\text {th }}$ International Conference on Applied Mechanics and Mechanical Engineering.

\title{
DETECTION OF HIDDEN CORROSION USING ULTRASONIC NONDESTRUCTIVE EVALUATION
}

HRAIRI $^{*}$ M. and KHAMIS ${ }^{* *} \mathrm{H}$.

\begin{abstract}
Hidden corrosion detection is a serious problem in many industries and in the aerospace industry in particular. Initiating on the inside or in the interface of an aircraft's skin, the corrosion must be tested from the outside surface. Ultrasonic bulk waves are often used in thickness detection of plate structures by measuring the time-of-flight. An experimental study of hidden corrosion detection by using ultrasonic non-destructive evaluation (NDE) in the laboratory is presented. Corroded metal as well as corrosion simulation samples were used as specimen for investigation. Normal beam probe was used for testing and qualitative results based on the A-scan oscilloscope display outputs were obtained.
\end{abstract}

\section{KEY WORDS}

Nondestructive evaluation, ultrasonic, A-scan, hidden corrosion, steel, aluminum

* Assistant professor, Dept. of Mechanical Engineering, IIUM, Kuala Lumpur, Malaysia

** Graduate student, Dept. of Mechanical Engineering, IIUM, Kuala Lumpur, Malaysia 


\section{INTRODUCTION}

Paint coatings used on the outer skin of metals function primarily as a protective measure against environmental effects for the underlying structure. However, over time there is a possibility for corrosion to occur underneath the coating especially for aging structures. Corrosion under paint is classified as hidden damage on structures as these damages are not visible to the eye and often require the stripping of paint as well as the complete disassembly of structures. These rather aggressive maintenance procedures are needed to ensure that hidden damage is not present in structural components, which could compromise the structural integrity of the component if left unchecked. These actions are time-consuming and inefficient. Hence, the need to reduce maintenance requirements for routine paint coating removal operations in support of condition based maintenance programs.

Numerous methods have been developed and tested for a wide variety of hidden damage detection problems. Many of these methods involve extensions and improvements to the traditional five nondestructive evaluation methods: ultrasound, eddy current, magnetic penetrant, fluorescent penetrant, and radiography. In particular, the following question arises: "Does an NDE capability exist that can accomplish through-coating inspections with the same effectiveness of current visual inspection methods where the coating system has been removed?".

Ultrasonic NDE is one of the most commonly used and oldest techniques for assessing the structural integrity of a part, and for detecting cracks and corrosion. It involves the insertion of mechanical vibration energy into a material substrate, which propagates as elastic waves through the material. The internal structure of the host material can be assessed by monitoring how the elastic waves propagate through the material, and by characterizing any elastic scattering events that are caused, for example, by localized cracks and corrosion. Both contact and noncontact approaches have been used to insert and detect the mechanical energy into the material substrate, with piezoelectric transducers being the overwhelming source and receiver elements in traditional ultrasonic NDE systems. Noncontact ultrasound measurement approaches utilizing aircouple transducers, electromechanical acoustic transducers (EMATs), and laser-based ultrasound have also shown significant promise and progress recently [1].

Contact transducers typically require a coupling fluid to efficiently transfer the mechanical energy into the structure. Raster-scanned versions of each of the ultrasound approaches described above provide area inspection coverage, but are time consuming, somewhat complicated to setup and operate, and typically require a highly trained inspector. Laser-based systems are also typically expensive and somewhat fragile.

Resolution and sensitivity levels have a strong dependence on the frequency and energy levels used, along with the type of analysis processing that is done. Corrosion material loss level sensitivities of $5-10 \%$ have been reported with spatial resolutions approaching 10 s of microns for megahertz transducer frequencies [2]. Laser ultrasound measurements are typically optical diffraction limited (1-5 microns) for spatial resolution limits which are due to the use of a focused laser beam as the probe. Material loss level measurements have recently reported below $1 \%$ in lap-joint specimens [3]. 
The possibility of making full-field measurements using ultrasound energy has also recently been reported by Lasser et. al. [4], and Bar-Cohen et. al. [5]. By combining ultrasonic array transducer designs with real-time, charge-coupled device technologies, the possibility of imaging ultrasound inspections has become a possibility. The devices operate in a similar to a camcorder device, providing framerate (30 fps) measurement capabilities. The size of the inspection area is still rather small $(\sim 1 \mathrm{~cm} 2)$, but the possibility of making whole-field, wide-area ultrasound inspections through coatings is very appealing.

Some of the primary disadvantages of ultrasound inspections include:

- Typically requires the use of a couplant medium

- Data sets can be large, ambiguous, and complex

- Coupling/adhesion is needed between the coating and the substrate material

- Difficult to use with complex geometry structures

- Raster-scanning of ultrasound transducer is typically required to acquire whole-field images which is time consuming, expensive, complex, and limits system/measurement portability

The objective of this paper is to investigate whether the ultrasonic NDE can accomplish through-paint inspections of corrosion. An understanding of the NDE methods in theory is necessary for the results' interpretation of this experiment. In completion of this study, it is aimed that the aforementioned arising question can be answered.

\section{EXPERIMENTAL PROCEDURE}

\section{Test Specimens}

Elastic waves propagating in solid plates with free boundaries are dispersive in nature. The waveforms and phase velocities along the plates as well as the displacement and stress distributions along the plate sections are dependent on the frequency and the plate thickness. Because of the frequency-thickness dependence of wave modes, there is a possibility of detecting corrosion or erosion thinning of structures with ultrasound waves. In particular, at least two types of features can be considered in the testing: (1) the time delay; and (2) transmission and reflection amplitudes. All of these methods offer the potential to inspect corrosion-induced thinning in structures, such as aircraft skin, chemical tanks, piping, ship hulls, and any other plate- or shell-like structure.

Two $5 \mathrm{~mm}$ thick of mild steel corrosion specimens have been used in the ultrasonic NDE experimental work:

1. A corroded sample, that was divided into quadrants for inspection purposes, in order to compare the intensity of corrosion on the surface as detected by the non-destructive testing method.

2. A second plate, without corrosion on its surface, served as a reference specimen.

Both plates were later sprayed with black nonconductive paint. Fig. 1 shows the corroded specimen before painting and the quadrants division while Fig. 2 shows the control specimen before painting. 


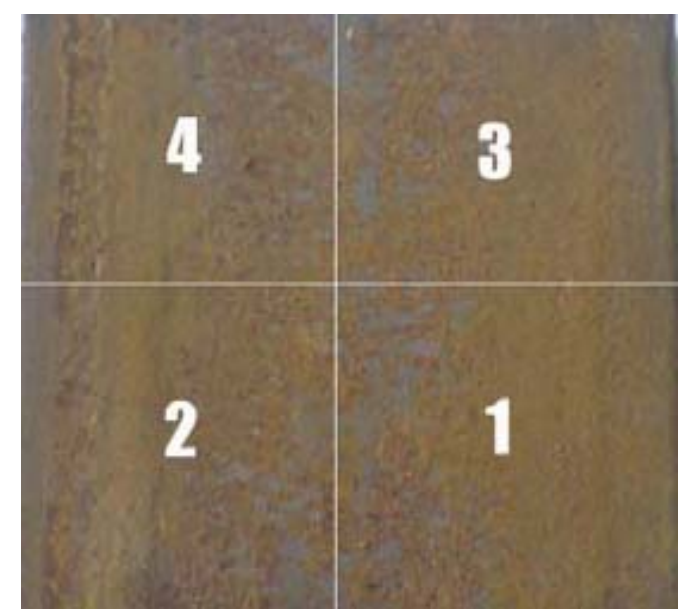

Fig. 1. Corroded specimen divided into quadrants

As can be seen from Fig. 1, the intensity of corrosion differs among the quadrants and pitting had occurred in quadrants 2 and 4 . The corroded sample was part of metal sheet kept inside the Engineering Workshop store room where the temperature is at room temperature and a possibility of moderate to high moisture content in air.

The reference specimen has no visible corrosion on its surface. Placed in the same room as the corroded sample, the reference specimen had only been in storage for a few days therefore corrosion had not occurred on the surface.

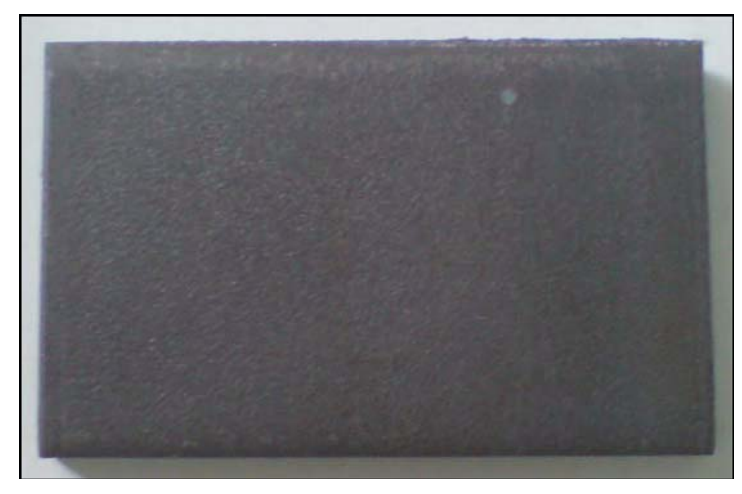

Fig. 2. Specimen without corrosion on the surface

\section{NDT Instrumentation}

The ultrasonic NDE instrumentation used in this experiment consisted of a TDScan ${ }^{\circledR}$ Ultrasonic Tester, a normal beam transducer and an ultrasound gel. The ultrasonic tester is capable to display a single A-Scan on its display output. A-scan is the ultrasonic echo amplitude response as a function of time.

Before initiating the inspection on the test specimen, the Ultrasonic Tester was calibrated using the calibration block. The probe used was a normal transverse beam (beam angle of $0^{\circ}$ ) at a frequency of $5 \mathrm{MHz}$. The speed of the beam was adjusted to $5950 \mathrm{~m} / \mathrm{s}$, consistent with the indicated acoustic properties of mild steel. A layer of 
couplant gel was applied on the specimens and the probe was placed on the specimens for readings. The resulting oscilloscope display for the reference specimen and each of the quadrants was then recorded for the results' interpretation stage of the experiment.

\section{RESULTS AND DISCUSSIONS}

The basic working principle of an ultrasound pulse-echo test consists of a transducer that sends out a pulse of energy and the same transducer listens for reflected energy (an echo). Reflections occur due to the presence of discontinuities and the surfaces of the test article. The amount of reflected sound energy is displayed versus time, which provides the inspector information about the size and the location of features that reflect the ultrasound wave. Fig. 3 shows a typical response of a corroded sample using a single A-scan of the UT tester.

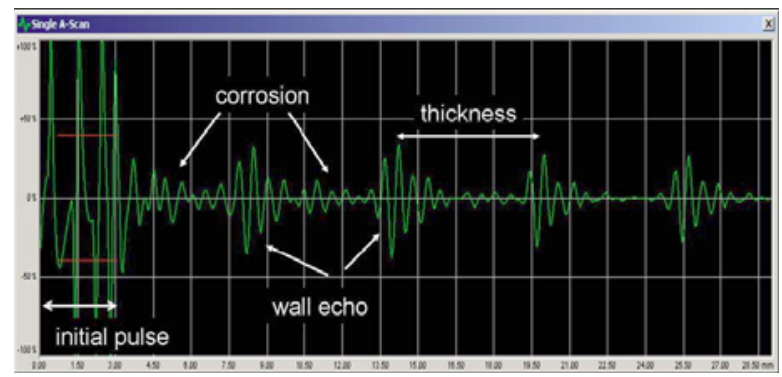

Fig. 3. Single A-scan display output of the oscilloscope on the specimen surface

As seen from Fig. 3, the first $3 \mathrm{~mm}$ of the display is considered as the initial pulse of the ultrasonic wave as it passes from the transducer and into the material's surface. A back wall echo is produced when the ultrasonic wave reaches the back wall of the material and is reflected back to the transducer. This is due to the inability of the ultrasonic wave to travel through air. The time/distance between the back wall echo and the initial pulse represents the thickness of the material which could be calculated using the following simple equation:

$$
d=\frac{v t}{2}
$$

where $d$ is the material thickness, $v$ is the ultrasound velocity and $t$ is the time between front wall and back wall echoes.

If a flaw is present in the material, some of the wave energy is reflected thus producing a defect echo between the initial pulse and the wall echo. A straight line indicates a flawless specimen. This can be observed in Fig. 4, where the output displays from the non-corroded and the corroded samples are compared.

It should be noted that the defect echo from the corrosion are broad and weak as compared to the back wall echo. This feature enables the distinction between a corrosion defect echo and the wall echo. A comparison between the intensity of corrosion on the quadrants of the corroded specimen can be observed in Fig. 5. It can be seen that the corrosion intensity is higher in quadrants 2 and 4 compared to 
quadrants 1 and 3 . Judging against the condition of the corroded sample (Fig. 1), pitting corrosion had occurred on quadrant 2 and 4; therefore the results obtained from the pulse-echo test reflect the condition of the sample.

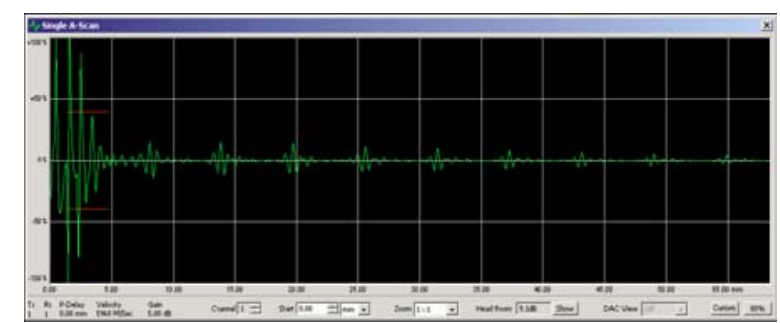

(a) Non-corroded specimen

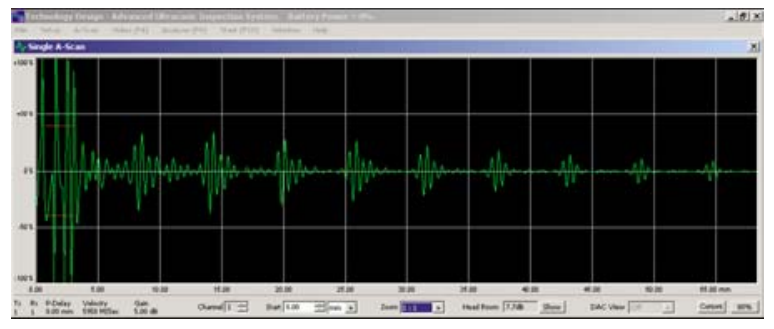

(b) Corroded sample at Quadrant 2

Fig. 4. A-scan display of non-corroded control specimen and corroded sample

The below results showed that measurement taken by a single NDE technique is not fully reliable and very often incomplete due to the operating range and limitation which characterize the sensor or the inspection technique. The use of multiple NDE has numerous advantages over single sensor inspection technique. Reduction in inspection time, increase reliability, improved defect visualization, and information redundancy are the benefits in using multiple NDE sensor information.

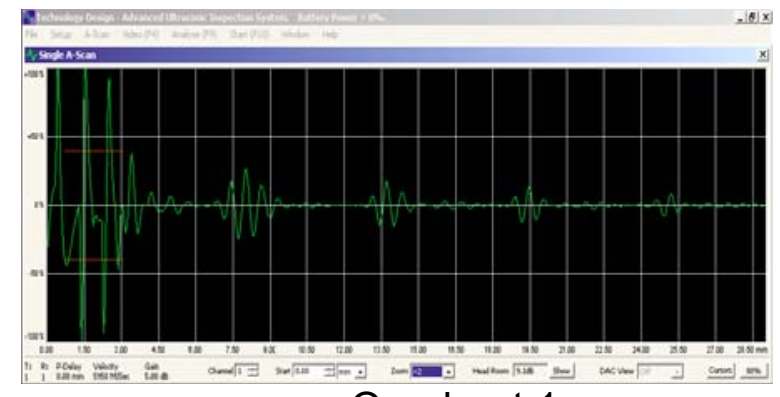

Quadrant 1

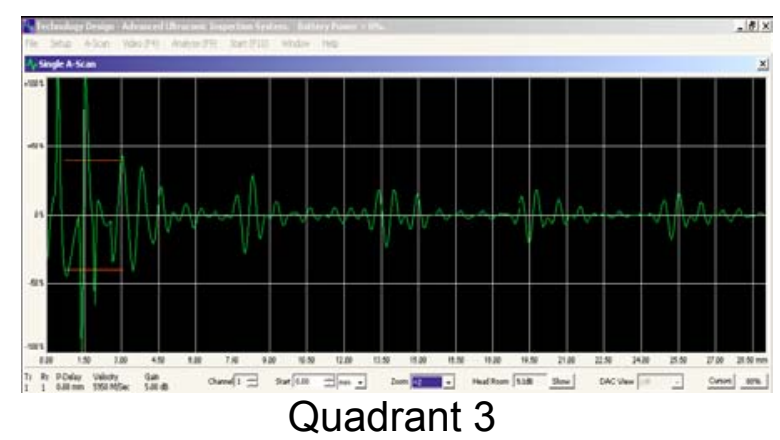

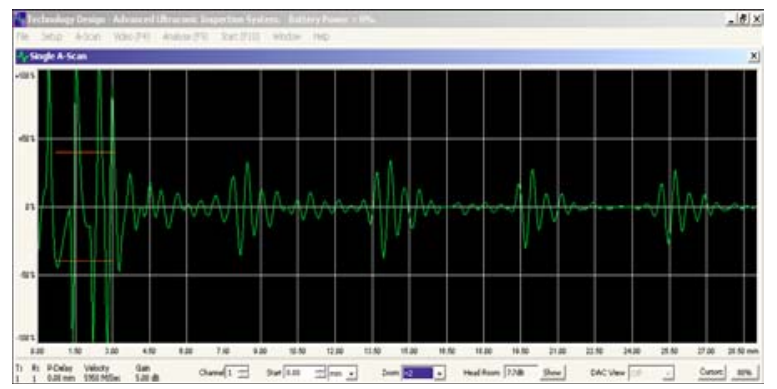

Quadrant 2

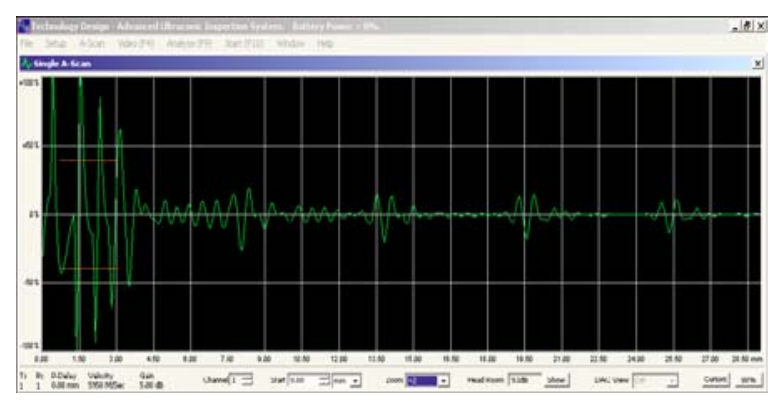

Quadrant 4

Fig. 5. Display outputs for the quadrants of the corroded sample

In interpreting the results, the thickness of the paint was not taken into account. Therefore the corrosion intensity was determined by qualitative analysis and not quantitatively as the thickness of the paint is unknown. Therefore corrosion sizing cannot be carried out due to the lack of sensitivity and time-base corrections when 
calibrating the ultrasonic tester. The absence of these adjustments to sensitivity and time base is because the calibration block is bare metal and not painted. However, as the objective of the project is to detect corrosion under paint it is sufficient to have a qualitative analysis for the results' interpretations.

Limiting the use of transducers to the normal transverse beam only is another limitation to the analysis of the results. According to the NORDTEST method, generally the angle beam probe scanning is used for detection of corrosion areas while the straight beam probe is applied for corrosion sizing. If both the angle beam and straight beam probes were used, better results hence better analysis could be obtained.

Another limitation to the results analysis is the lack of C-scan feature on the Ultrasonic Tester that could enable a corrosion mapping to be performed. Corrosion mapping will allow locating the corroded areas on the specimen under the paint and thus, producing a better quality analysis. It is important to note that ample amount of couplant medium is required to obtain better data displays as insufficient amount will lead to lesser transmission of ultrasonic waves.

\section{CONCLUSION}

Although there were limitations that need to be rectified, Ultrasonic NDE can be used to detect hidden corrosion under paint. A-scan enables depth measurement thus indicating the loss of thickness of the material due to corrosion and by qualitative analysis the intensity of corrosion can be determined.

Further improvements to this simple analysis can be carried out by combining different NDE techniques or incorporating the use of an angle beam probe along the normal beam measurements.

The aim of the project is to answer to the arising question of: "Does an NDE capability exists that can accomplish through-coating inspections with the same effectiveness of current visual inspection methods where the coating system has been removed?" Based on the results obtained, this study answers "yes" to the said question.

\section{REFERENCES}

[1] Tuzzeo, D., "Noncontact Air-Coupled Guided Wave Ultrasonics for Detection of Thinning Defects in Aluminum Plates," Research in NDE, vol. 13, no. 2, (2001).

[2] Blackshire, J.L., Buynak, C., Steffes, G., and Marshall, R., "Nondestructive Evaluation through Aircraft Coatings: A State- of- the- Art Assessment", 9th Joint FAA/DoD/NASA Conference on Aging Aircraft, (2006)

[3] Choquet, M., Levesque, D., Massabki, M., Neron, C., Bellinger, N., Forsyth, D., Chapman, C., Gould, R., Komorowski, J., and Monchalin, J., "Laser-Ultrasonic Detection of Hidden Corrosion in Aircraft Lap Joints," Review of Progress in Quantitative Nondestructive Evaluation, Vol. 20B, pp. 300-307, (2001)

[4] Lasser, M., Lasser, B., Kula, J., and Rohrer, G., "Developments in Real-Time 2D Ultrasound Inspection for Aging Aircraft", SPIE Conference on Nondestructive Evaluation of Aging Aircraft, Airports, and Aerospace Hardware III, Vol. 3586, pp. 78-84, (1999). 
[5] Bar-Cohen, Y., Mal, A., and Lasser, M., "NDE of Hidden Flaws in Aging Aircraft Structures using Obliquely Backscattered Ultrasound Signals (OBUS)," SPIE Conference on Nondestructive Evaluation of Aging Aircraft, Airports, and Aerospace Hardware III, Vol. 3586, pp. 347-353, (1999).

[6] Chahbaz, A., Gauthier, J., Brassard M., and Hay, R., "Ultrasonic Techniques for Hidden Corrosion Detection in Aircraft Wing Skin", Third Joint DoD/FAA/NASA conference on Aging Aircraft, (1999).

[7] Zhu, W., Rose, J.L., Barshinger, J.N., and Agarwala, V.S., "Ultrasonic Guided Wave NDT for Hidden Corrosion Detection“, Res Nondestr Eval, vol. 10, no. 4, pp. 205-225, (1998). 